\title{
Qualidade dos cuidados de enfermagem: contribuições de enfermeiros especialistas em enfermagem médico-cirúrgica
}

\author{
Quality of nursing care: contributions from expert nurses in medical-surgical nursing
}

Como citar este artigo:

Ribeiro OMPL, Martins MMFPS, Sousa PAF, Trindade LL, Forte ECN, Silva JMAV. Quality of nursing care: contributions from expert nurses in medical-surgical nursing. Rev Rene. 2020;21:e43167. DOI: https://doi.org/10.15253/2175-6783.20202143167

(D) Olga Maria Pimenta Lopes Ribeiro ${ }^{1}$
(D) Maria Manuela Ferreira Pereira da Silva Martins ${ }^{1}$
(1) Paulino Artur Ferreira de Sousa ${ }^{1}$
(D) Letícia de Lima Trindade ${ }^{2}$
(DElaine Cristina Novatzki Forte ${ }^{3}$
(D) João Miguel Almeida Ventura da Silva ${ }^{1}$

${ }^{1}$ Escola Superior de Enfermagem do Porto. Porto, Portugal.

${ }^{2}$ Universidade do Estado de Santa Catarina. Chapecó, SC, Brasil.

${ }^{3}$ Universidade Federal de Santa Catarina.

Florianópolis, SC, Brasil.

\section{Autor correspondente:}

Olga Maria Pimenta Lopes Ribeiro

Escola Superior de Enfermagem do Porto

Rua Dr. António Bernardino de Almeida

4200-072. Porto, Portugal.

E-mail: olgaribeiro@esenf.pt

\begin{abstract}
RESUMO
Objetivo: identificar a realização de atividades congruentes com os padrões de qualidade dos cuidados de enfermagem por enfermeiros especialistas em enfermagem médico-cirúrgica, no contexto hospitalar. Métodos: estudo descritivo, concretizado em 36 hospitais, no qual participaram 259 enfermeiros especialistas em enfermagem médico-cirúrgica. Para coleta de dados, usou-se de questionário, posteriormente analisado por meio de estatística descritiva. Resultados: os enfermeiros participantes avaliaram com melhores escores as atividades relativas às dimensões responsabilidade e rigor, satisfação do cliente e prevenção de complicações; e com menores escores, as atividades integradas nas dimensões promoção da saúde, readaptação funcional e bem-estar e autocuidado. Conclusão: embora os achados evidenciem atuação dos enfermeiros especialistas na área de enfermagem médico-cirúrgica que tende a ser coerente com os padrões de qualidade, sinalizaram-se lacunas no exercício profissional e potencialidades que podem subsidiar o processo de qualificação desses profissionais, alertar os gestores e qualificar a assistência de enfermagem.

Descritores: Cuidados de Enfermagem; Especialidades de Enfermagem; Garantia da Qualidade dos Cuidados de Saúde; Enfermagem; Hospitais.
\end{abstract}

\footnotetext{
ABSTRACT

Objective: to identify the performance of activities congruent with the quality standards of nursing care by expert nurses in medical-surgical nursing, in the hospital context. Methods: a descriptive study, carried out in 36 hospitals, in which 259 nurses specialized in medical-surgical nursing participated. For data collection, a questionnaire was used, later analyzed using descriptive statistics. Results: the participating nurses evaluated activities related to the dimensions of responsibility and rigor, customer satisfaction and prevention of complications with better scores; and with lower scores, activities integrated in the dimensions of health promotion, functional readaptation and well-being and self-care. Conclusion: although the findings show the performance of expert nurses in the medical-surgical nursing area that tends to be consistent with quality standards, gaps in professional practice and potentialities that can support the qualification process of these professionals were signaled, alert managers and qualify nursing care.

Descriptors: Nursing Care; Specialties, Nursing; Quality Assurance, Health Care; Nursing; Hospitals.
} 


\section{Introdução}

A nível mundial, as alterações sociais, políticas e econômicas têm modificado o contexto de trabalho, especialmente no domínio da saúde, tornando-o mais complexo e diversificado, o que tem exigido dos profissionais competências altamente diferenciadas que culminem em maior eficácia e eficiência, no âmbito da conceção e implementação dos cuidados ${ }^{(1)}$. As últimas décadas registram mudanças importantes nas necessidades de saúde das populações. Tais alterações têm desafiado os atuais sistemas de saúde em todo o mundo, exigindo cuidados de qualidade adequados às reais necessidades das pessoas, responsabilidade que não pode ser cumprida sem a garantia da qualidade da assistência de enfermagem ${ }^{(2)}$.

A existência de profissionais de enfermagem, em número significativo, em todas as unidades de saúde, determina que a qualidade da assistência de enfermagem seja uma das componentes da qualidade em saúde ${ }^{(3)}$. Os enfermeiros, enquanto prestadores de cuidados constituem a base para segurança e qualidade dos cuidados, sendo que o empoderamento e o envolvimento deles resultarão em melhor desempenho e maior qualidade em saúde ${ }^{(4)}$.

Acrescente-se, ainda, que regulação do exercício profissional é fator determinante na procura da qualidade, em Portugal. Assim, em 2001, a Ordem dos Enfermeiros teve como desafio a criação de padrões de qualidade que, visando explicitar os diversos aspectos do papel social da enfermagem, constituem para enfermeiros orientação para prática profissional de excelência ${ }^{(5)}$. Na medida em que as percepções desses profissionais sobre a qualidade podem constituir-se em indicadores válidos da qualidade global dos cuidados, assim como da segurança dos clientes, no contexto hospitalar ${ }^{(6)}$, a compreensão do envolvimento de enfermeiros na realização de atividades coerentes com os padrões de qualidade torna-se imperativo fundamental.

Em relação ao delineamento dos padrões de qualidade dos cuidados de enfermagem, disponibili- zaram-se seis categorias: a organização dos cuidados de enfermagem, a readaptação funcional, o bem-estar e autocuidado, a prevenção de complicações, a promoção da saúde, bem como a satisfação do cliente $^{(5)}$. Em seguida, em cada área de especialização, definiram-se os padrões de qualidade relativos aos cuidados especializados, com propósito de servirem de referenciais para prática especializada. No caso da especialidade em enfermagem médico-cirúrgica, considerando a abrangência desta, definiram-se, recentemente, quatro áreas: enfermagem à pessoa em situação crítica, à pessoa em situação paliativa, à pessoa em situação perioperatória e à pessoa em situação crônica ${ }^{(7)}$. De modo a atender a esta recente alteração, houve necessidade de reformular os padrões de qualidade dirigidos aos cuidados especializados em enfermagem médico-cirúrgica ${ }^{(8)}$. Todavia, independente dessas reformulações, permanecem na base da atuação de enfermeiros os padrões de qualidade dos cuidados de enfermagem ${ }^{(5)}$.

Assim, esta pesquisa partiu do questionamento: como enfermeiros especialistas em enfermagem médico-cirúrgica, de instituições hospitalares de Portugal, expressam a concretização dos padrões de qualidade dos cuidados de enfermagem? Neste sentido, a investigação objetivou identificar a realização de atividades congruentes com os padrões de qualidade dos cuidados de enfermagem por enfermeiros especialistas em enfermagem médico-cirúrgica, no contexto hospitalar.

\section{Métodos}

Estudo descritivo, concretizado em 36 hospitais, enquadrados no modelo de gestão de Entidade Pública Empresarial. Para definição dos participantes, estabeleceu-se amostragem não probabilística por conveniência ${ }^{(9)}$. Definiram-se como critérios de inclusão: ser enfermeiro especialista na área de enfermagem médico-cirúrgica e exercer atividade profissional no contexto hospitalar, em período igual ou superior a seis meses, em unidades de medicina (clínica médica, 
no Brasil), cirurgia ou medicina intensiva e urgência. Dentre os enfermeiros elegíveis nos cenários que autorizaram o estudo, alcançou-se amostra de 259 enfermeiros, o que correspondeu a $35,4 \%$ da população estudada.

O instrumento de coleta de dados usado foi um questionário, dividido em duas partes, uma contendo as variáveis sociodemográficas e profissionais dos enfermeiros especialistas na área de enfermagem médico-cirúrgica e, outra, composta pela escala de percepção das atividades de enfermagem que contribuem para qualidade dos cuidados. De acordo com o referencial adotado, ou seja, os padrões de qualidade dos cuidados de enfermagem, essa parte do instrumento estava estruturada em sete dimensões, a saber: a satisfação do cliente, a promoção da saúde e a prevenção de complicações com três itens, o bem-estar e autocuidado e a readaptação funcional com quatro itens, a organização dos cuidados de enfermagem com dois itens e a responsabilidade e rigor com seis ${ }^{(10)}$. Em escala tipo Likert, a frequência de realização das atividades que contribuem para qualidade dos cuidados variava entre 1 e 4 , sendo que 1 referiu-se a nunca, 2 poucas vezes, 3 às vezes e 4 sempre.

A coleta de dados decorreu entre julho de 2015 e janeiro de 2016. Nesse momento, os questionários foram pessoalmente entregues pelas pesquisadoras aos participantes, que após preenchimento, colocaram-no individualmente em envelope, tendo sido posteriormente reunidos e tabulados. Para o tratamento dos dados, aplicou-se o software Statistical Package for the Social Sciences, versão 22.0, tendo sido realizadas análises estatísticas descritivas.

Importa referir que esta pesquisa seguiu os preceitos éticos orientadores para pesquisas em Portugal, estando, ainda, integrada em investigação mais abrangente, concretizada no território nacional, intitulada: "Os contextos da prática hospitalar e as concepções dos enfermeiros", sendo aprovada, inicialmente, pela Comissão de Ética para a Saúde de um Centro Hospitalar da Região Norte de Portugal, conforme número 98-
15. Os participantes, além de terem assinado termo de consentimento informado, não foram identificados em nenhuma etapa do estudo e autorizaram o uso de informações coletadas.

\section{Resultados}

Os 259 enfermeiros especialistas, na área de enfermagem médico-cirúrgica que participaram do estudo, eram majoritariamente do sexo feminino (70,3\%), com idade média de 39 anos, desvio de padrão de 7,6. Relativamente ao grau acadêmico, 57,5\% possuiam licenciatura, $41,7 \%$ mestrado, seguindo-se do doutorado e bacharelado (0,4\% em ambas). Dos 108 enfermeiros com mestrado, 64,8\% eram mestres na área de especialidade investigada. No que concerne à distribuição dos enfermeiros participantes, segundo as regiões da Administração Regional de Saúde em que se inserem os hospitais, prevaleceu o Norte $(57,9 \%)$, seguindo-se o Centro $(23,9 \%)$, Lisboa e Vale do Tejo (13,1\%), o Alentejo (4,2\%) e o Algarve $(0,8 \%)$. Em relação aos contextos onde exerciam a atividade, prevaleceram as unidades de medicina $(45,5 \%)$, medicina intensiva e urgência $(44,1 \%)$ e, por fim, cirurgia $(10,4 \%)$. No que se refere à atividade profissional na área de especialização, o tempo médio foi 4,1 anos (com um desvio de padrão de 5,2). No concernente à formação sobre os padrões de qualidade, 132 enfermeiros especialistas $(51,0 \%)$ declararam que a concretizaram.

Decorrente da utilização da escala de percepção das atividades de enfermagem que contribuem para qualidade dos cuidados, a Tabela 1 ilustra os achados referentes às sete dimensões analisadas. Com relação à satisfação do cliente, no âmbito do respeito pelos valores, pelas crenças, pelas capacidades e pelos desejos dos clientes, bem como da procura de empatia nas interações estabelecidas com os pacientes, a resposta sempre foi majoritária. Por outro lado, o envolvimento das pessoas significativas do cliente, no desenvolvimento do processo de cuidados, foi a ati- 
vidade que registrou menor valor percentual no sempre. Além disso, 7,3\% dos participantes referiram que poucas vezes concretizavam a referida atividade.

No que se refere à promoção da saúde, o reconhecimento das condições de saúde, bem como dos recursos dos clientes, o incentivo, durante a internação, à adoção de estilos de vida saudáveis, bem como a transmissão de informação que potencialize a aprendizagem cognitiva e a aquisição de novas capacidades pelos clientes, constituíram atividades que os participantes realizavam, majoritariamente, às vezes. Destaca-se, ainda, que $14,3 \%$ dos enfermeiros especialistas em enfermagem médico-cirúrgica referiram que poucas vezes aproveitam a internação para incentivar os clientes a adotarem estilos de vida saudáveis.

Em relação à prevenção de complicações, o reconhecimento de potenciais problemas nos clientes, a prescrição e execução de intervenções para prevenir as complicações, bem como a avaliação das intervenções que permitem evitar os problemas ou diminuir as consequências indesejáveis, foram atividades em que os participantes responderam, a maioria, sempre.

No bem-estar e autocuidado, o reconhecimento dos problemas dos clientes neste domínio, a prescrição e execução de intervenções que maximizem o bem-estar e a realização das atividades de vida pelos clientes, a avaliação das intervenções implementadas, bem como a referenciação de situações problemáticas, constituem atividades pontualmente concretizadas pelos participantes. De fato, nas quatro atividades integradas no enunciado descritivo bem-estar e autocuidado, a resposta às vezes foi a mais frequente.

A respeito da dimensão readaptação funcional, os participantes apenas realizavam majoritariamente sempre a atividade que se referia à continuidade do processo de cuidados de enfermagem. No planejamento da alta dos clientes em função das necessidades e dos recursos existentes na comunidade, na otimização das capacidades dos clientes e das pessoas significativas para gerirem o regime terapêutico, bem como no ensino, na instrução e no treinamento dos clientes, tendo em vista a adaptação individual, os enfermeiros especialistas referiram realizar, em maioria, às vezes essas atividades. Aponta-se, ainda, que $13 \mathrm{a}$ $20,0 \%$ dos participantes realizavam poucas vezes essas três atividades.

Na dimensão organização dos cuidados de enfermagem, quando questionados se dominavam o sistema de registros, a resposta predominante foi sempre $(63,3 \%)$. Ainda assim, aproxidamente, $37,0 \%$ dos enfermeiros participantes referiram não dominar em todas as situações o sistema de registros de enfermagem. Em relação ao conhecimento que tinham sobre as políticas do hospital, a resposta mais prevalente foi às vezes $(48,6 \%)$.

No que reporta à dimensão responsabilidade e rigor, na evidência de responsabilidade nas decisões que toma e nos atos que pratica e delega, tendo em vista a prevenção de complicações e, por outro lado, o bem-estar e autocuidado, a resposta sempre foi a mais prevalente. Ainda nessa dimensão, quando questionados sobre o rigor técnico e científico na implementação de intervenções, no âmbito da prevenção de complicações, bem como no âmbito do bem-estar e autocuidado, os participantes continuaram a responder, predominantemente, sempre. Salienta-se que nas atividades relativas à referenciação de problemas em outros profissionais, bem como na supervisão das atividades integradas nas intervenções de enfermagem e nas atividades delegadas, embora a resposta majoritária tenha sido sempre, os valores percentuais foram menores do que nas outras atividades dessa dimensão.

Para finalizar, importa destacar que embora em todas as dimensões apresentadas na Tabela 1, as respostas às vezes e sempre tenham predominado, foram as dimensões promoção da saúde e readaptação funcional que obtiveram maior número de enfermeiros que realizavam poucas vezes as respectivas atividades. Por sua vez, as atividades mais realizadas foram as integradas nas dimensões responsabilidade e rigor e satisfação do cliente. 
Tabela 1 - Distribuição das respostas referentes às atividades de enfermagem que contribuem para qualidade dos cuidados. Portugal, 2016

\begin{tabular}{|c|c|c|c|c|}
\hline \multirow{2}{*}{ Dimensões } & \multirow{2}{*}{$\begin{array}{l}\text { Nunca } \\
\mathbf{n}(\%)\end{array}$} & \multirow{2}{*}{$\begin{array}{c}\begin{array}{c}\text { Poucas } \\
\text { vezes }\end{array} \\
n(\%)\end{array}$} & \multirow{2}{*}{$\begin{array}{c}\text { Às vezes } \\
\mathbf{n}(\%)\end{array}$} & \multirow{2}{*}{$\begin{array}{c}\text { Sempre } \\
\mathbf{n}(\%)\end{array}$} \\
\hline & & & & \\
\hline \multicolumn{5}{|l|}{ Satisfação do Cliente } \\
\hline Atua respeitando os valores, as crenças, as capacidades, bem como os desejos dos clientes & - & $2(0,8)$ & $75(28,9)$ & $182(70,3)$ \\
\hline Procura empatia nas interações que estabelece com os clientes & - & $0(0,0)$ & $75(29,0)$ & $184(71,0)$ \\
\hline Envolve as pessoas significativas do cliente no desenvolvimento do processo de cuidados & - & $19(7,3)$ & $144(55,6)$ & $96(37,1)$ \\
\hline \multicolumn{5}{|l|}{ Promoção da Saúde } \\
\hline Reconhece as condições de saúde, bem como os recursos dos clientes & - & $22(8,5)$ & $160(61,8)$ & $77(29,7)$ \\
\hline Incentiva durante a internação a adoção de estilos de vida saudáveis & - & $37(14,3)$ & $134(51,7)$ & $88(34,0)$ \\
\hline $\begin{array}{l}\text { Transmite informação que potencializa a aprendizagem cognitiva e a aquisição de novas } \\
\text { capacidades pelos clientes }\end{array}$ & - & $24(9,3)$ & $147(56,8)$ & $88(33,9)$ \\
\hline \multicolumn{5}{|l|}{ Prevenção de Complicações } \\
\hline Reconhece potenciais problemas nos clientes & - & $2(0,8)$ & $107(41,3)$ & $150(57,9)$ \\
\hline Prescreve e executa intervenções para prevenir complicações & - & $3(1,2)$ & $105(40,5)$ & $151(58,3)$ \\
\hline $\begin{array}{l}\text { Realiza a avaliação das intervenções, de modo a evitar os problemas ou diminuir as } \\
\text { consequências indesejáveis }\end{array}$ & - & $12(4,6)$ & $103(39,8)$ & $144(55,6)$ \\
\hline \multicolumn{5}{|l|}{ Bem-estar e Autocuidado } \\
\hline Reconhece os problemas dos clientes no âmbito do bem-estar e autocuidado & - & $12(4,6)$ & $127(49,1)$ & $120(46,3)$ \\
\hline $\begin{array}{l}\text { Prescreve e executa intervenções que maximizem o bem-estar, assim como a realização das } \\
\text { atividades de vida pelos clientes }\end{array}$ & - & $10(3,9)$ & $146(56,3)$ & $103(39,8)$ \\
\hline Faz a avaliação das intervenções implementadas & - & $16(6,2)$ & $146(56,4)$ & $97(37,4)$ \\
\hline Referencia situações problemáticas & - & $13(5,0)$ & $140(54,1)$ & $106(40,9)$ \\
\hline \multicolumn{5}{|l|}{ Readaptação Funcional } \\
\hline Atua na continuidade do processo de cuidados de enfermagem & $1(0,4)$ & $17(6,6)$ & $103(39,8)$ & $138(53,2)$ \\
\hline Planeja a alta dos clientes em função das necessidades e dos recursos existentes na comunidade & $2(0,8)$ & $52(20,1)$ & $123(47,4)$ & $82(31,7)$ \\
\hline Otimiza as capacidades dos clientes e pessoas significativas para gestão do regime terapêutico & $4(1,5)$ & $36(13,9)$ & $125(48,3)$ & $94(36,3)$ \\
\hline Ensina, instrui e treina os clientes, tendo em vista a adaptação individual & - & $39(15,1)$ & $137(52,9)$ & $83(32,0)$ \\
\hline \multicolumn{5}{|l|}{ Organização dos Cuidados de Enfermagem } \\
\hline Demonstra domínio sobre o sistema de registros de enfermagem & - & $13(5,0)$ & $82(31,7)$ & $164(63,3)$ \\
\hline Tem conhecimento sobre as políticas da instituição hospitalar & $2(0,8)$ & $17(6,6)$ & $126(48,6)$ & $114(44,0)$ \\
\hline \multicolumn{5}{|l|}{ Responsabilidade e Rigor } \\
\hline $\begin{array}{l}\text { Tendo em vista prevenir complicações, evidencia responsabilidade nas decisões que toma e nos } \\
\text { atos que pratica e delega }\end{array}$ & - & - & $18(6,9)$ & $241(93,1)$ \\
\hline $\begin{array}{l}\text { Tendo em vista o bem-estar e autocuidado, evidencia responsabilidade nas decisões que toma } \\
\text { e nos atos que pratica e delega }\end{array}$ & - & - & $28(10,8)$ & $231(89,2)$ \\
\hline $\begin{array}{l}\text { No âmbito da prevenção de complicações, implementa as intervenções com rigor técnico e } \\
\text { científico }\end{array}$ & - & - & $31(12,0)$ & $228(88,0)$ \\
\hline $\begin{array}{l}\text { No âmbito do bem-estar e autocuidado, implementa as intervenções com rigor técnico e } \\
\text { científico }\end{array}$ & - & $1(0,4)$ & $47(18,1)$ & $211(81,5)$ \\
\hline Faz referenciação dos problemas para outros profissionais & - & $6(2,3)$ & $91(35,1)$ & $162(62,6)$ \\
\hline $\begin{array}{l}\text { Faz supervisão das atividades integradas nas intervenções de enfermagem, bem como das } \\
\text { atividades delegadas }\end{array}$ & - & $12(4,6)$ & $79(30,5)$ & $168(64,9)$ \\
\hline
\end{tabular}




\section{Discussão}

Neste estudo, assumem-se como limitações a amostragem por conveniência, que impede a generalização dos resultados, bem como a resistência de enfermeiros do contexto hospitalar em participar de investigações e, consequentemente, de responder a mais um instrumento de coleta de dados.

Todavia, por meio da utilização da escala das atividades de enfermagem que contribuem para qualidade dos cuidados ${ }^{(10)}$, identificou-se a contribuição dos enfermeiros especialistas, na área de enfermagem médico-cirúgica, para qualidade da assistência de enfermagem. Enfatiza-se que o conhecimento sobre as atividades mais e menos realizadas por estes profissionais, alertará os diferentes níveis de gestão para o direcionamento necessário, em prol do desenvolvimento da profissão, bem como de estratégias a serem adotadas, no sentido de melhorar a concretização dos padrões de qualidade.

O impacto do envolvimento de enfermeiros na qualidade, segurança e experiência de cuidado tem tido especial enfoque nos últimos anos. Neste sentido, o triplo objetivo de promover a saúde das pessoas, reduzir o custo e melhorar a experiência de cuidado do cliente e do enfermeiro, somente poderão ser alcançados com força de trabalho robusta e vinculada, que encontre significado na atividade profissional ${ }^{(11)}$, e se empenhe permanentemente em melhorar a qualidade da assistência de enfermagem.

A Ordem dos Enfermeiros, em 2005, com intuito de promover a implementação de sistemas de melhoria contínua da qualidade em enfermagem, realizou, em diversos locais do país, sessões de formação sobre os padrões de qualidade, o que justifica o fato de mais da metade dos enfermeiros especialistas, na área de enfermagem médico-cirúrgica, afirmarem terem a referida formação. Em estudo semelhante a este, realizado com 306 enfermeiros especialistas na área de enfermagem de reabilitação, a porcentagem de participantes que realizou a formação foi idêntica ${ }^{(12)}$.

Quanto à concretização das atividades, no âm- bito da satisfação do cliente, os resultados denotaram que apesar da evidência relativamente ao envolvimento da pessoa significativa no desenvolvimento do processo de cuidados, na prática dos enfermeiros e enfermeiros especialistas, nem sempre se garante essa interação, o que efetivamente também foi confirmado em outros estudos ${ }^{(12-13)}$.

No que se refere à promoção da saúde, as respostas apontaram para realização pontual das três atividades incluídas nesse enunciado descritivo, o que esteve em consonância com o obtido por outros autores $^{(12-14)}$. Estes achados revelaram que embora teoricamente esteja definida a importância da ajuda profissional dos enfermeiros na mudança de comportamentos, por meio de abordagens centradas na pessoa e no potencial desta ${ }^{(8,15)}$, as práticas dirigidas à promoção da saúde não foram ainda convenientemente reconhecidas e integradas por enfermeiros ${ }^{(13)} \mathrm{e}$ enfermeiros especialistas ${ }^{(12)}$, em hospitais.

Em relação à prevenção de complicações, os resultados comprovam a importância da prática profissional centrada na detecção precoce de problemas e na prevenção de complicações. Estes resultados, para além de corroborarem outros estudos que também utilizaram o mesmo instrumento de coleta de $\operatorname{dados}^{(12-13)}$, são singulares, por traduzirem os aspectos centrais da área de especialidade em enfermagem médico-cirúrgica, bem como a influência, ainda, notória do modelo biomédico flexneriano e do referencial teórico de Florence Nightingale. Em estudo realizado em Portugal ${ }^{(16)}, 60$ a 70,0\% dos enfermeiros consideraram estar de acordo ou totalmente de acordo com a prática, as concepções de pessoa, saúde, ambiente e enfermagem de Florence Nightingale, o que efetivamente terá favorecido modelos assistenciais centrados na prevenção de complicações.

0 bem-estar e autocuidado constituem a dimensão em que os resultados obtidos diferiram de estudo realizado com enfermeiros especialistas na área de enfermagem de reabilitação ${ }^{(12)}$ que, neste caso, é o único estudo semelhante em que os dados podem ser comparados. De fato, enquanto os enfermeiros espe- 
cialistas em enfermagem médico-cirúrgica sinalizaram, em maioria, às vezes, quanto às quatro atividades do enunciado descritivo bem-estar e autocuidado, os enfermeiros especialistas na área de enfermagem de reabilitação referiram reconhecer sempre os problemas dos clientes, bem como prescrever, implementar e avaliar majoritariamente sempre as intervenções que culminem na maximização do bem-estar e na realização das atividades de vida pelos clientes ${ }^{(12)}$.

Resultados idênticos foram encontrados na dimensão readaptação funcional. Neste caso, enquanto os enfermeiros especialistas na área de enfermagem médico-cirúrgica mencionaram realização pontual das atividades, os enfermeiros especialistas em reabilitação referiram majoritariamente oferecer sempre continuidade ao processo de cuidados e, pelo menos, metade declararam proceder sempre às atividades de planejar a alta dos clientes, de acordo com as necessidades destes e os recursos existentes na comunidade; otimizar as capacidades dos clientes e pessoas significativas para gestão do regime terapêutico; e ensinar, instruir e treinar os clientes, tendo em vista a adaptação individual ${ }^{(12)}$.

Atualmente, perante a exigência de orientação disciplinar que se pretende, cada vez mais clara ${ }^{(17)}$, o paradigma na enfermagem, foca-se, essencialmente, nas capacidades de cada pessoa, na promoção do potencial desta para adaptação às várias transições vivenciadas ao longo do ciclo vital, na possibilidade de incorporar novos conhecimentos e adquirir novas habilidades $^{(8,14,16)}$, bem como na ajuda profissional de enfermeiros, no sentido de promover, ao máximo, a autonomia da pessoa. Assim, os resultados obtidos no bem-estar e autocuidado e readaptação funcional continuam a alertar para possível dicotomia entre a teoria exposta e o evidenciado nas práticas ${ }^{(13)}$, constituindo duas áreas em que é necessário investir.

Em estudo realizado em Portugal, com intuito de identificar os referenciais teóricos que mais se adequam à prática dos enfermeiros no contexto hospitalar, os resultados evidenciaram que a teoria das necessidades de Virginia Henderson continua a orientar o exercício profissional dos enfermeiros portugueses. Logo, salienta-se que, com base neste referencial, a tendência é identificar os problemas e as necessidades dos clientes e planejar intervenções focadas na substituição da pessoa, muitas vezes, em detrimento de intervenção mais centrada na promoção do autocuidado e na própria readaptação funcional(16).

Além do mencionado, atendendo à relevância de todas as fases do processo de enfermagem, importa que para além da identificação das necessidades e dos problemas dos clientes, os enfermeiros especialistas invistam na prescrição, implementação e avaliação das intervenções que tenham em vista para resolução, garantindo a continuidade dos cuidados, a otimização das capacidades dos clientes e a melhor preparação para alta.

Na dimensão organização dos cuidados de enfermagem, o fato dos enfermeiros não dominarem em todas as situações o sistema de registros, muitas vezes, por desconhecerem as atualizações efetuadas nos mesmos, poderá ter implicações na produção de indicadores de qualidade. Em relação ao conhecimento sobre as políticas do hospital, a resposta mais prevalente foi às vezes, o que corrobora resultados apresentados por outros autores ${ }^{(12-13)}$.

No que reporta à dimensão responsabilidade e rigor, enfatiza-se que, embora nos estudos realizados anteriormente $^{(12-13)}$, os participantes sinalizassem majoritariamente sempre todas as atividades, os valores percentuais constatados no caso dos enfermeiros especialistas na área de enfermagem médico-cirúrgica foram mais elevados, denotando maior percepção de responsabilidade e rigor por parte destes profissionais. Em consonância com resultados de outros autores $^{(12-13)}$, ainda no âmbito da dimensão responsabilidade e rigor, referenciar problemas que estão para além das competências e supervisionar as atividades integradas nas intervenções de enfermagem, bem como as atividades delegadas, foram aspectos que os enfermeiros perceberam como menos concretizados, existindo, portanto, nestas áreas, oportunidade de melhoria e maiores investimentos. 


\section{Conclusão}

Os resultados evidenciaram atuação dos enfermeiros especialistas na área de enfermagem médico-cirúrgica, tendencialmente, congruente com os padrões de qualidade. Da análise efetuada, as atividades mais realizadas reportaram-se às dimensões responsabilidade e rigor, satisfação do cliente e prevenção de complicações. Por outro lado, as atividades integradas nas dimensões promoção da saúde, readaptação funcional e bem-estar e autocuidado foram aquelas que os enfermeiros especialistas perceberam como menos executadas, exigindo, portanto, que a este nível, sejam repensadas as práticas, de modo a culminar em atuação coerente com as referidas dimensões.

\section{Colaborações}

Ribeiro OMPL contribuiu com concepção e projeto, análise e interpretação dos dados e redação do artigo. Martins MMFPS colaborou com concepção e projeto e aprovação final da versão a ser publicada. Sousa PAF auxiliou na revisão crítica relevante do conteúdo intelectual e aprovação final da versão a ser publicada. Trindade LL, Forte ECN e Silva JMAV contribuíram com interpretação dos dados e redação do artigo.

\section{Referências}

1. Silva RM, Luz MD, Fernandes JD, Silva LS, Cordeiro $\mathrm{AL}$, Mota LS. Becoming a specialist: Portuguese nurses' expectations after completing the specialization program. Rev Ref. 2018; 4(16):147-54. doi: https://doi.org/10.12707/RIV17076

2. Gaalan K, Kunaviktikul W, Akkadechanunt T, Wichaikhum OA, Turale S. Factors predicting quality of nursing care among nurses in tertiary care hospitals in Mongolia. Int Nurs Rev. 2019; 66(2):176-82. doi: https://doi.org/10.1111/ inr.12502

3. Nilsson J, Johansson E, Egmar AC, Florin J, Leksell J, Lepp M, et al. Development and validation of a new tool measuring nurses self-reported profes- sional competence - The nurse professional competence (NPC) Scale. Nurse Educ Today. 2014; 34(4):574-80. doi: https://doi.org/10.1016/j. nedt.2013.07.016

4. Wei H, Sewell KA, Woody G, Rose MA. The state of the science of nurse work environments in the United States: a systematic review. Int J Nurs Sci. 2018; 5(3):287-300. doi: https://doi. org/10.1016/j.ijnss.2018.04.010

5. Ordem dos Enfermeiros (PT). Padrões de qualidade dos cuidados de enfermagem, enquadramento conceptual. Enunciados descritivos [Internet]. 2012 [citado 2020 jan 13]. Disponível em: https://www.ordemenfermeiros.pt/media/8903/ divulgar-padroes-de-qualidade-dos-cuidados.pdf

6. Smeds-Alenius L, Tishelman C, Lindqvist $\mathrm{R}$, Runesdotter S, McHugh M. RN assessments of excellent quality of care and patient safety are associated with significantly lower odds of 30day inpatient mortality: A national cross-sectional study of acute-care hospitals. Int J Nurs Stud. 2016; 61:117-24. doi: https://doi.org/10.1016/j. ijnurstu.2016.06.005

7. Ordem dos Enfermeiros (PT). Regulamento $\mathrm{n}^{\mathrm{o}}$ 429/2018 de 16 de junho. Diário da República no 135, II Série [Internet]. 2018 [citado 2020 jan 13]. Disponível em: https://dre.pt/home/-/ dre/115698617/details/maximized

8. Ordem dos Enfermeiros (PT). Padrões de qualidade dos cuidados especializados em enfermagem médico-cirúrgica [Internet]. 2017 [citado 2020 jan 13]. Disponível em: https://www.ordemenfermeiros.pt/media/5681/ponto-2_padroes-qualidade-emc_rev.pdf

9. Coutinho CP. Metodologia de investigação em ciências sociais e humanas: teoria e prática. Coimbra: Almedina; 2014.

10. Martins MM, Gonçalves MN, Ribeiro OM, Tronchin DM. Quality of nursing care: instrument development and validation. Rev Bras Enferm. 2016; 69(5):864-70. doi: http://dx.doi. org/10.1590/0034-7167-2015-0151

11. Dempsey C, Assi MJ. The impact of nurse engagement on quality, safety, and the experience of care: what nurse leaders should know. Nurs Adm Q. 2018; 42(3):278-83. doi: http://dx.doi. org/10.1097/NAQ.0000000000000305 
12. Martins MM, Ribeiro OM, Silva JM. The contribution of nurses specialists in rehabilitation nursing to quality of care. Rev Portug Enferm Reabil [Internet]. 2018 [cited Jan 18, 2020]; 1(1):22-9. Available from: https://www.aper.pt/ficheiros/ revista/rperv1n1.pdf

13. Ribeiro OM, Martins MM, Tronchin DM. Nursing care quality: a study carried out in Portuguese hospitals. Referência. 2017; 4(14):89-100. doi: http://dx.doi.org/10.12707/RIV16086

14. Freire RM, Lumini MJ, Martins MM, Martins T, Peres HHC. Taking a look to promoting health and complications' prevention: differences by context. Rev Latino-am Enfermagem. 2016; 24:e2749. doi: http://dx.doi.org/10.1590/15188345.0860.2749
15. International Council of Nurses. Nurses: a force for change - care effective, cost effective. Genebra: International Council of Nurses; 2015.

16. Ribeiro OM, Martins MM, Tronchin DM, Forte EC. The perspective of Portuguese nurses on nursing metaparadigmatic concepts. Texto Contexto Enferm. 2018; 27(2):e3970016. doi: http://dx.doi. org/10.1590/0104-070720180003970016

17. Watson J. Clarifying the discipline of nursing as foundational to development of professional nursing [editorial]. Texto Contexto Enferm. 2017; 26(4). doi: http://dx.doi.org/10.1590/010407072017002017 editorial4

\section{(cc) BY}

Este é um artigo de acesso aberto distribuído sob os termos da Licença Creative Commons 